Acta Math. Hungar., 127 (3) (2010), 220-224.

DOI: $10.1007 / \mathrm{s} 10474-010-9120-\mathrm{z}$

First published online March 18, 2010

\title{
SEMIMODULAR LATTICES AND THE HALL-DILWORTH GLUING CONSTRUCTION
}

\author{
E. T. SCHMIDT* \\ Mathematical Institute of the Budapest University of Technology and Economics, \\ Müegyetem rkp. 3, H-1521 Budapest, Hungary \\ e-mail: schmidt@math.bme.hu \\ URL: http://www.math.bme.hu/ schmidt/
}

(Received May 22, 2009; revised November 3, 2009; accepted November 12, 2009)

\begin{abstract}
We present a new gluing construction for semimodular lattices, related to the Hall-Dilworth construction.
\end{abstract}

The gluing constructions in the lattice theory started with a paper of M. Hall and R. P. Dilworth [4] to prove that there exists a modular lattice that cannot be embedded in any complemented modular lattice. This construction is the following: let $K$ and $L$ be lattices, let $F$ be a filter of $K$, and let $I$ be an ideal of $L$ such that $F$ and $I$ are isomorphic with $\varphi: F \rightarrow I$. Then we form the disjoint union $G=K \cup L$ and identify $a \in F$ with $a \varphi \in I$, for all $a \in F . a \leqq b$ in $G$ iff one of the following cases is satisfied: (i) $a \leqq_{K} b$, $a, b \in K$, (ii) $a \leqq_{L} b, a, b \in L$, (iii) $a \leqq_{K} z$ and $z \varphi \leqq_{L} b, a \in K, b \in L$ for some $z \in F$.

I applied in my paper [5] the following special gluing construction (to give a very short proof for the theorem that every semimodular lattice of finite length has a cover-preserving embedding into a simple semimodular lattice), which is shown in Fig. 1. We define this gluing.

Let $L$ and $K$ be semimodular lattices of finite length. Take a maximal chain $C$ of $L$. Assume that $K$ contains a filter $C^{\prime}$ isomorphic to $C$ under the isomorphism $\psi: C \rightarrow C^{\prime}$. Consider the attachment $G$ of the lattice $K$ to the lattice $L$ over $C$ by identifying $C$ with $C^{\prime}$ along $\psi$, in the sense of G. Grätzer

\footnotetext{
${ }^{*}$ This research was supported by the Hungarian National Foundation for Scientific Research (OTKA), grant no. K 77432 .

Key words and phrases: lattice, semimodular, gluing.

2000 Mathematics Subject Classification: primary 06C10, secondary 06B15.
}

0236-5294/\$20.00 @ 2010 Akadémiai Kiadó, Budapest 


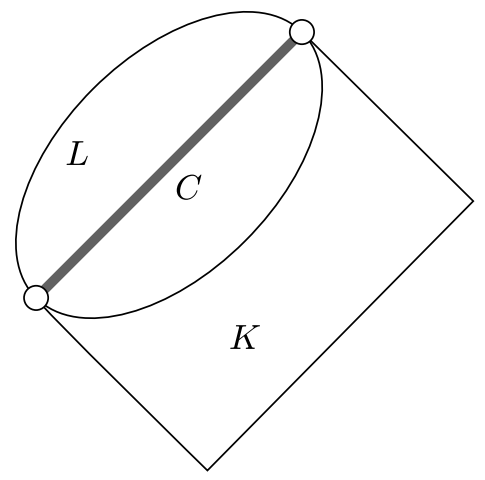

Fig. 1: The sketch of the gluing $G=L \cup_{C} K$

and D. Kelly [2]. So we have $G=L \cup K$ with $L \cap K=C$ and the order

$$
a \leqq b= \begin{cases}a \leqq_{K} b & \text { if } a, b \in K \\ a \leqq_{L} b & \text { if } a, b \in L \\ a \leqq_{K} x \psi, x \leqq_{L} b & \text { if } a \in K, b \in L \text {, for some } x \in C .\end{cases}
$$

We denote the lattice thus obtained by $G=L \cup_{C} K$. This lattice is evidently not a Hall-Dilworth gluing. The maximal chain $C$ is not an ideal of $L$, but this construction reminds of the Hall-Dilworth gluing.

Despite this we will prove that by the given construction the HallDilworth gluing is in the 'background'. This makes it possible to introduce a new gluing construction for semimodular lattices.

A join-homomorphism $\varphi: L \rightarrow K$ is said to be cover-preserving iff it preserves the relation $\preceq$. Similarly, a join-congruence $\Phi$ of $L$ is called coverpreserving if the natural join-homomorphism $L \rightarrow L / \Phi, x \mapsto[x] \Phi$ is coverpreserving. The image of a semimodular lattice by a cover-preserving joinhomomorphism is a semimodular lattice (see [3]).

TheOREM. $G=L \cup_{C} K$ is the cover-preserving join-homomorphic image of a semimodular lattice $H, \psi: H \rightarrow G$ such that:

(1) $H$ is the Hall-Dilworth gluing of the semimodular lattices $P$ and $K$ over a chain isomorphic to $C$,

(2) $P$ is the direct power of a chain isomorphic to $C$,

(3) $L$ is the cover-preserving join-homomorphic image of $P, \varphi: P \rightarrow L$,

(4) the restriction of $\psi$ to the filter $P$ of $H$ is $\varphi$ and to $K$ is the identity.

Proof. We have the semimodular lattices $L$ and $K$ of finite length. $C$ is a maximal chain of $L$ and $K$ contains $C^{\prime}$ as filter. Further, $L \cap K=C$ and $G=L \cup_{C} K$ is the glued semimodular lattice.

In [1], we have proved (see Theorem 1): each finite semimodular lattice $L$ is a cover-preserving join-homomorphic image of the direct product 
of $\mathrm{w}(J(L))$ finite chains (the width $\mathrm{w}(P)$ of a (finite) poset $P$ is defined to be $\max \{n: P$ has an $n$-element antichain $\})$. The outline the proof is: let $k=\mathrm{w}(J(L)) . J(L)$ is the union of $k$ appropriate chains. Let us extend these chains of $J(L)$ to maximal chains $C_{1}, \ldots, C_{k}$ of $L$. Then $J(L)$ $\subseteq C_{1} \cup \cdots \cup C_{k}$. We may assume that $k \geqq 2$. Denote $C_{1} \times \cdots \times C_{k}$ by $P$ and define a join-homomorphism

$$
\varphi: P \rightarrow L, \quad\left(x_{1}, \ldots, x_{k}\right) \mapsto x_{1} \vee \cdots \vee x_{k} .
$$

This proves (3). The maximal chains $C_{i}$ are all isomorphic to $C$ which means that (2) is satisfied, $P \cong C^{k}$.

Observe that to represent $L$ as a cover-preserving join-homomorphic image of a direct product of chains we use only that $J(L) \subseteq C_{1} \cup \cdots \cup C_{k}$ and therefore we may assume that $k \geqq \mathrm{w}(J(L))$ and one of the maximal chains in the definition of $P$ is the selected $C \subset L, C=C_{k}$. We will regard $C_{k}$ as the ideal $I$ of $P$ in the usual manner. On the other hand, in $K$ we have the filter $C^{\prime}$. Take the Hall-Dilworth gluing of $P$ and $K$ by identifying $I$ and $C^{\prime}$. We get obviously a semimodular lattice $H=P \cup K$ and condition (1) is satisfied.

Finally, we define $\psi: H \rightarrow G$. Denote $\Phi$ the join congruence of $P$ induced by $\varphi$. By the definition $\varphi: P \rightarrow L(0,0, \ldots, 0, c) \varphi=c$ and therefore if $c, c^{\prime} \in C, c \neq c^{\prime}$ we have $(0,0, \ldots, 0, c) \neq\left(0,0, \ldots, 0, c^{\prime}\right)(\Phi)$. We define $\Psi$ on $H$ such that the restriction of $\Psi$ to the filter $P$ is $\Phi$ and the restriction to $K$ is the trivial join congruence.

A sublattice $\left\{a_{1} \wedge a_{2}, a_{1}, a_{2}, a_{1} \vee a_{2}\right\}$ of a lattice is called a covering square if $a_{1} \wedge a_{2} \prec a_{i} \prec a_{1} \vee a_{2}$ for $i=1,2$.

In [1] we proved the following characterization of cover-preserving join congruences: let $\Phi$ be a join-congruence of a finite semimodular lattice $M$. Then $\Phi$ is cover-preserving if and only if for any covering square $S=\{a \wedge$ $b, a, b, a \vee b\}$ if $a \wedge b \not \equiv a(\Phi)$ and $a \wedge b \not \equiv b(\Phi)$ then $a \equiv a \vee b(\Phi)$ implies $b \equiv a \vee b(\Phi)$. Using this result we can see that $\Psi$ is a cover-preserving join congruence of $H$. (4) is obviously satisfied.

Obviously, $(1,1, \ldots, 1,1)=(0,0, \ldots, 0,1)(\Phi)$, consequently, the image of $I \subset H$ is the maximal $C \subset G$.

We illustrate the proof with the following easy example. $L$ is the well known seven element semimodular lattice $N_{7}$, and $K$ is an arbitrary semimodular lattice with a filter which is a chain of length 3 .

$P$ is isomorphic to $C^{2}$ and $\varphi$ is a cover-preserving join homomorphism. The image of all elements in $A$ is the unit element.

From $P$ and $K$ we get the following lattice $H$ with the Hall-Dilworth gluing:

In the class of all semimodular lattices we introduce a natural generalization of the Hall-Dilworth gluing: 

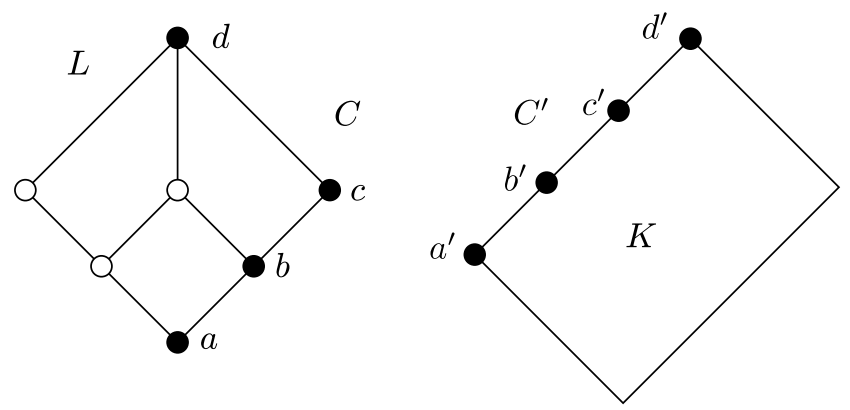

Fig. 2: The lattices $L$ and $K$ with the chains $C, C^{\prime}$

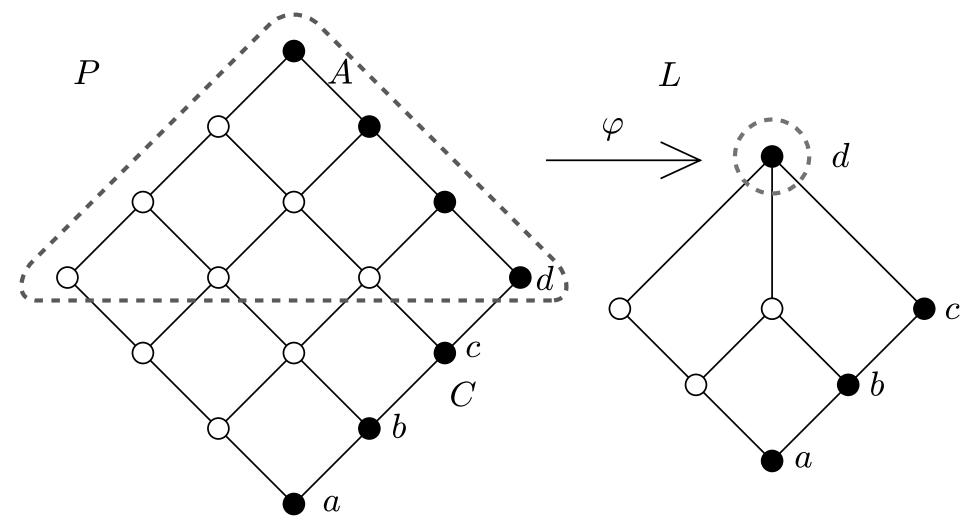

Fig. 3

Definition. Let $K$ and $L$ be semimodular lattices, $F$ a filter of $K$, and $I$ an ideal of $L$. If $F$ is isomorphic to $I$ with $\varphi$ the isomorphism, then we can form first the lattice $G$, the Hall-Dilworth gluing of $L$ and $K$ over $F$ and $I$. Let $\Phi$ be a cover-preserving join-congruence of $G$. We call $G / \Phi$ the gluing of $L / \Phi$ and $K / \Phi$.

$L / \Phi$ is in general only a cover-preserving sublattice of $G / \Phi$. In our special case the restriction of $\Phi$ to $K$ is the trivial join congruence $\omega$. 


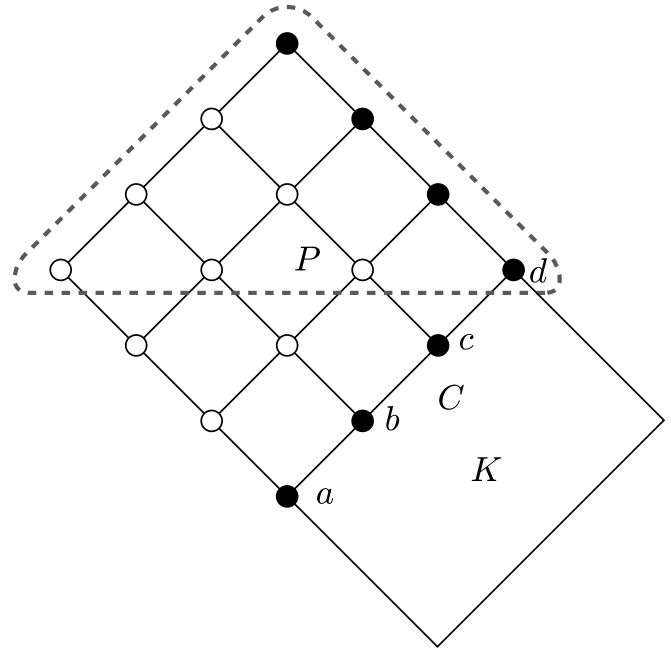

Fig. 4: $H=P \cup K$ with $C$

\section{References}

[1] G. Czédli and E. T. Schmidt, How to derive finite semimodular lattices from distributive lattices?, Acta Math. Hungar., 121 (2009), 277-282.

[2] G. Grätzer and D. Kelly, On congruence lattices of $m$-complete lattices, Algebra Universalis, 53 (2005), 253-265.

[3] G. Grätzer and E. Knapp, Notes on planar semimodular lattices. I. Construction, Acta Sci. Math. (Szeged), 73 (2007), 445-462.

[4] M. Hall and R. P. Dilworth, The embedding problem for modular lattices, Annals of Math., 45 (1944), 450-456.

[5] E. T. Schmidt, Cover-preserving embeddings of finite semimodular lattices into simple semimodular lattices, Algebra Universalis, 61 (2009), http://www.math.bme.hu/ schmidt/publist.htm 